\title{
The Optimum Rate of Inter-Frequency Scan in Inter-Frequency HetNets
}

\author{
Ali Mahbas, Huiling Zhu and Jiangzhou Wang, \\ School of Engineering and Digital Arts, University of Kent, Canterbury, CT2 7NT, United Kingdom \\ Email: \{ajm83, H.Zhu, J.Z.Wang \}@kent.ac.uk
}

\begin{abstract}
Inter-frequency scan (IFS) is a process carried out by the terminals to discover the small cells (SCs) in the interfrequency heterogeneous networks (HetNets) prior to offload them to the discovered SCs. The IFS has a great impact on the quality of service, the energy efficiency and the spectral efficiency in the cellular systems. In this paper, a framework is presented to model and evaluate the impact of IFS on the system performance by using the stochastic geometry. The energy efficiency is derived as performance metric to obtain the optimum value of the IFS rate (optimum number of scans per unit time) by taking into consideration the trade-off in the offloading process between the power consumption and exploiting the system resources efficiently. Considering the energy consumption for performing IFSs along with the energy consumption for maintaining the uplink transmission will help to find the optimum value of IFS rate that achieves the best energy efficiency. The analysis and results show that the optimum IFS rate depends on different system parameters such as SCs' density, terminal's speed and the transmit power of the SCs (SCs' coverage).
\end{abstract}

\section{INTRODUCTION}

The inter-frequency deployment, where a dedicated frequency channel (e.g. high frequency) is used at the small cells (SCs), has been adopted to overcome the limitation of frequency bandwidth in the current cellular systems and to meet the high data rate requirements. Since the SCs are deployed on a different frequency from the macro cells (MCs), a SC detection process is essential to offload traffic from the overloaded MCs to the SCs in the network [1], [2]. The SC detection process in the inter-frequency deployment is defined as the inter-frequency scans (IFSs) carried out by the terminals on the high frequency periodically, in order to establish whether these terminals are in the SCs' coverage prior to begin offloading them to the near SCs. However, there is a trade-off between exploiting the available high frequency resources and the power consumption at the terminals [2], [3]. Unnecessary power consumption takes place when the UEs perform scans more frequently, or the terminals miss the SCs' coverage significantly and keep connecting to the MCs with limited resources when they perform IFSs infrequently. In other words, in order to save the power of terminals' batteries, they need to reduce the number of IFSs per unit time. However, small number of IFSs may cause failure in offloading more traffic from MCs to the SCs which results in less spectral efficiency and overloading the MCs. Therefore, finding the optimum IFS rate will improve the system performance significantly in the current cellular systems.

Some work has been done to propose new mechanisms to tackle this issue. [3] proposed a new SC structure by reusing the low frequency at each high frequency SC to form a doublelayer SC. In this approach, the terminals do not need to perform IFS periodically which save significant power at the terminals with no trade-off in offloading to the SCs, however, this approach may require some changes in the current system design. A few schemes have been proposed such as mobility status based scheme and radio fingerprint scheme [4], [5]. In the mobility status based scheme, the terminals are triggered to reduce the number of IFSs when moving fast. However, the slow terminals still need to perform a big number of IFSs per unit time, which cost these terminals very large power consumption. At the same time, the radio fingerprint schemes require a large memory at the terminals' side and significant amount of signalling to maintain and update this memory. In [6], stochastic geometry was used to investigate the impact of IFSs on the average energy efficiency by approximating the offloading loss as a liner function of the IFS rate $\left(s_{g}\right)$. This approximation ignores the impact of all other parameters and our analysis shows that offloading loss is not only affected by $s_{g}$, but also affected by other parameters such as the density of SCs, the terminal's speed and the coverage and distribution of the small cells around the terminal's path. It is also assumed in this paper that the fraction of a typical terminal's path covered by the SCs is obtained by multiplying the tier association with the total time of transition.

The main contribution in this paper is to model the impact of periodic IFS on the system performance by taking into consideration the effect of different system parameters including the transmit power of the small cells, the density of small cells and the speed of the terminals. Stochastic geometry is used to model the locations of SCs and to investigate the coverage of SCs on a typical terminal's path. A framework is also presented to obtain the optimum IFS rate that achieves the best system performance in a two-tier HetNet. The energy efficiency is derived as a performance metric by taking into consideration the trade-off between the energy consumption and the efficient utilization of the system resources.

The rest of this paper is structured as follows: Section II describes the system model. The offloading loss is addressed in Section III. In Section IV, energy efficiency is defined. In Section V, the optimum value of IFS rate is obtained. In Section VI, the system performance is shown by numerical simulation results. Conclusions are drawn in Section VII. 


\section{SySTEM MOdEL}

A two-tier heterogeneous network (HetNet) is considered in this paper where each tier uses $F_{k}$ frequency $k \in[1,2]$, as shown in Fig. 1. Both tiers are assumed to be distributed as Poisson point process (PPP), $\Phi_{k}$ with density $\lambda_{k}$. It is also assumed that $E_{s}$ is the total energy consumption at a terminal of interest (denoted by $\mathcal{T}_{0}$ ) for performing IFSs:

$$
E_{s}=N_{s} p_{s} t_{s}
$$

where $p_{s}$ represents the power required for one IFS, $t_{s}$ is the time that each IFS lasts and $N_{s}$ is the total number of IFSs that $\mathcal{T}_{0}$ performs per unit time. $N_{s}$ is a random variable which depends on the time that $\mathcal{T}_{0}$ camps on the MCs. In order to find $N_{s}$, the time that $\mathcal{T}_{0}$ camps on SCs and MCs needs to be investigated. We consider the random waypoint model (RWP) [7]. Assume that $\mathcal{T}_{0}$ chooses its starting point $\left(X_{0}\right)$ and destination point $\left(X_{1}\right)$ uniformly in the disc $\left(0, R_{\text {sys }}\right)$ where $R_{\text {sys }}$ represents the system radius. It is also assumed that $\mathcal{T}_{0}$ moves with $S$ speed and spends $T_{p}$ pause time at $X_{1}$. Therefore, the expected value of the distance between $X_{0}$ and $X_{1}$ can be obtained as:

$$
\mathbb{E}\left[D_{X_{0}-X_{1}}\right]=\mathbb{E}\left[\left\|X_{1}-X_{0}\right\|\right]=\frac{125 R_{\text {sys }}}{45 \pi}
$$

where $\|$.$\| indicates the Euclidean distance.$

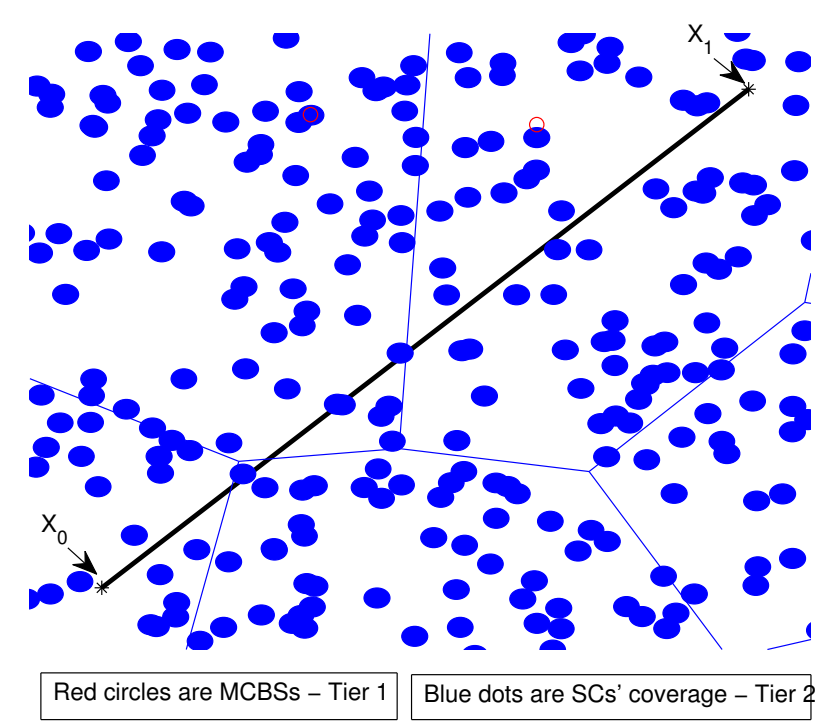

Fig. 1. Two-Tier Inter-Frequency HetNet

Since there is no interference between SCs and MCs, it is assumed that $\mathcal{T}_{0}$ camps on any $\mathrm{SC}$ when the received power from this $\mathrm{SC}$ satisfies the condition below:

$$
\rho_{0} \leq p_{r, 0} \geq \max _{i \in \Phi_{2}} p_{r, i}
$$

where $\rho_{0}$ is the minimum received power and $p_{r, i}$ is the received power from $i$ th $\mathrm{SC}$. According to this condition, the average coverage of each SC (fading is averaged out) forms a circle (including some of overlap areas). Since there is no interference from the first tier, a regular shape for cells has been assumed for finding the sojourn time and handover rate [8], [9].

It is assumed that all the SCs have the same transmit power and they have the same coverage. Since the SCs basestations (SCBSs) are uniformly distributed in the network as a PPP, the expected number of SCs that are crossed by $\mathcal{T}_{0}$ during its movement from $X_{0}$ to $X_{1}\left(M_{X_{0}-X_{1}}\right)$ can be expressed as:

$$
\mathbb{E}\left[N_{s c}\right]=2 R_{s c} \lambda_{2} \mathbb{E}\left[D_{X_{0}-X_{1}}\right]
$$

where $R_{s c}=\left(\frac{\rho_{o}}{p_{2} P L_{2}}\right)^{\frac{-1}{\alpha_{2}}}$ is the radii of each SC, $p_{2}$ and $\alpha_{2}$ are the transmit power and path-loss exponent of SCs respectively, $P L_{2}=\frac{c}{f_{2} 4 \pi}$ is the path-loss of the high frequency at 1 meter where $c$ is the velocity of the wave and $f_{2}$ is the high frequency used by the SCs, and $2 \mathbb{E}\left[D_{X_{0}-X_{1}}\right]\left(\frac{\rho_{o}}{p_{2} P L_{2}}\right)^{\frac{-1}{\alpha_{2}}}$ represents the area surrounding $M_{X_{0}-X_{1}}$. Any SC will be crossed by $\mathcal{T}_{0}$ if its SCBS is located in this area. The distance that $\mathcal{T}_{0}$ travels in the $i$ th SC that is located in the area and its SCBS is at distance $\nu_{i}$ from $M_{X_{0}-X_{1}}$ is shown in Fig. 2, and can be found as below:

$$
\bar{d}_{i}=2 R_{s c} \sin \left(\cos ^{-1}\left(\frac{\nu_{i}}{R_{s c}}\right) \quad \nu_{i} \leq R_{s c}\right.
$$

The coverage of $i$ th SC is a random variable, which depends on $\nu_{i}$. Since $\nu_{i}$ takes any value from 0 to $R_{s c}$, it is assumed that it has a uniform distribution in the range $\left[0, R_{s c}\right]$ with the probability density function (PDF) $\frac{1}{R_{s c}}$. The PDF of each coverage is obtained by using transforming density function:

$$
\begin{aligned}
f_{\bar{d}}(\bar{d}) & =f_{\nu}(\nu(\bar{d}))\left|\frac{d \nu}{d \bar{d}}\right| \\
& \stackrel{(a)}{=} \frac{1}{R_{s c}} \frac{d}{d \bar{d}}\left(R_{s c} \cos \left(\sin ^{-1}\left(\frac{\bar{d}}{2 R_{s c}}\right)\right)\right)
\end{aligned}
$$

where (a) is obtained from Eq. (5) and $\nu=$ $R_{s c} \cos \left(\sin ^{-1}\left(\frac{\bar{d}}{2 R_{s c}}\right)\right)$. Since the SCs locations are independent and from linearity exception, the expected value of the total SCs' coverage can be obtained by summing up the coverage of each $\mathrm{SC}$ crossed by $\mathcal{T}_{0}$ as shown:

$$
\begin{aligned}
\mathbb{E}\left[C_{\text {total }}\right] & =\mathbb{E}\left[N_{s c}\right] \int_{0}^{\infty} \bar{d} f_{\bar{d}}(\bar{d}) d \bar{d} \\
& =\mathbb{E}\left[N_{s c}\right] \int_{0}^{2 R_{s c}} \frac{\bar{d}^{2}}{4 r^{2} \sqrt{1-\frac{\bar{d}^{2}}{4 r^{2}}}} d \bar{d}
\end{aligned}
$$

where PDF of the coverage of each SC $f_{\bar{d}}(\bar{d})$ is found from solving Eq. (6) and the integral limits follow from the maximum and the minimum coverage of each SC being $2 R_{s c}$ and 0 respectively. Note that this includes some overlap coverage.

The expected value of the total time that $\mathcal{T}_{0}$ spends in the SC's coverage during $M_{X_{0}-X_{1}}$ can be expressed as

$$
\mathbb{E}\left[T_{s c}\right]=\frac{\mathbb{E}\left[C_{\text {total }}\right]}{S}+T_{p} \mathcal{A}_{s c}
$$




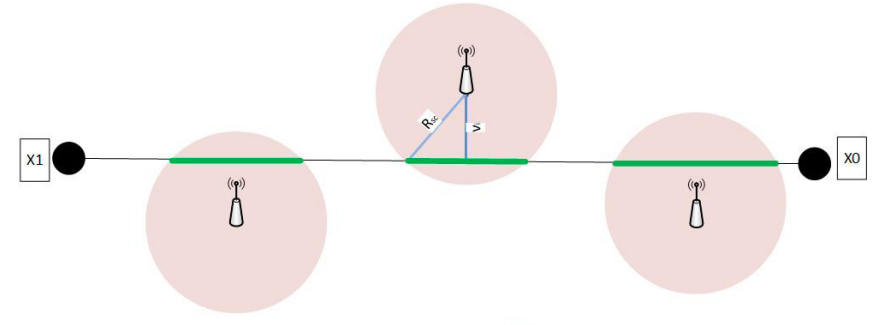

Small cells' coverage on the path Small cells' coverage

Fig. 2. Two-Tier Inter-Frequency HetNet

where $\mathbb{E}\left[C_{\text {total }}\right]$ is obtained in Eq. (7), $T_{p} \mathcal{A}_{s c}$ represents the expected time that $\mathcal{T}_{0}$ spends in any SC's coverage and $\mathcal{A}_{s c}$ is the probability that the destination point is located in the SCs' coverage. Since $\mathcal{T}_{0}$ is connected to the second tier when receiving $\rho_{0}$ from any SC as shown in Eq. (3), and the locations of SCBSs and the destination point are randomly distributed on the plane, $\mathcal{A}_{s c}$ can be found from null probability [10] and similar to [11]. Assume that the destination point $X_{1}$ is located at the origin, the probability that this point is in the SCs' coverage can be obtained as:

$$
\begin{aligned}
\mathcal{A}_{s c} & =1-\mathbb{P}\left[\max _{i \in \Phi_{2}} p_{r, i}<\rho_{0}\right] \\
& =1-\mathbb{P}\left[r_{0}>\left(\frac{\rho_{0}}{P L_{2} p_{2}}\right)^{\frac{-1}{\alpha_{2}}}\right] \\
& =1-\exp \left(-\pi \lambda_{2}\left(\frac{\rho_{0}}{P L_{2} p_{2}}\right)^{\frac{-2}{\alpha_{2}}}\right)
\end{aligned}
$$

where $r_{0}$ is the distance to the nearest SCBS.

\section{OFFLOADING LOSS}

The offloading loss is defined as the time that $\mathcal{T}_{0}$ spends in the SCs' coverage, but served by the first tier due to late SCs detection (when large value of $s_{g}$ is considered in the system). Therefore, there is a trade-off between the offloading loss and the power consumption in the periodic scan mechanism [2]. Finding the optimum value of $s_{g}$ can improve the system performance significantly. Since the SCs are randomly distributed around $\mathcal{T}_{0}$ 's path, the SCs can be grouped into two groups according to the time that $\mathcal{T}_{0}$ with speed $S$ travels in their coverage. $\mathcal{T}_{0}$ travels at least $s_{g}$ in each SC of the first group $\left(\Xi_{1}\right)$, therefore $\mathcal{T}_{0}$ misses these SCs' coverage partly from 0 to $s_{g}$ as it enters the coverage of each SC at a random time between two IFSs. The expected value of the time missed by $\mathcal{T}_{0}$ from each SC of $\Xi_{1}$ can be obtained as:

$$
\begin{aligned}
\mathbb{E}\left[T_{\Xi_{1}}\right] & =P_{\Xi_{1}} \int_{0}^{s_{g}} t_{1} f_{t_{1}}(t) d t \\
& =\left(1-\int_{0}^{\bar{d}_{s g}} f_{\bar{d}}(\bar{d}) d \bar{d}\right) \int_{0}^{s_{g}} \frac{t}{s_{g}} d t
\end{aligned}
$$

where $P_{\Xi_{1}}$ is the probability that any SC crossed by $\mathcal{T}_{0}$ belongs to $\Xi_{1}$, which can be also interpreted as the probability that $\mathcal{T}_{0}$ travels at least $\bar{d}_{s g}=S s_{g}$ in any SC's coverage. $f_{t_{1}}(t)$ is the PDF of the time that $\mathcal{T}_{0}$ misses from any $\mathrm{SC}$ of $\Xi_{1}$, and it is assumed to be uniformly distributed on the range $\left[0, s_{g}\right] . \mathcal{T}_{0}$ spends $t_{i}$ in $i$ th SC's coverage of $\Xi_{2}$, where $t_{i}<$ $s_{g}$. Therefore, it misses the coverage of these SCs partly or completely. The expected value of the time missed from a SC of $\Xi_{2}$ can be expressed as:

$$
\begin{aligned}
\mathbb{E}\left[T_{\Xi_{2}}\right] \stackrel{(a)}{=} \mathbb{E}_{t_{i}}\left(P_{\Xi_{2}}\left(\left(1-\frac{t_{i}}{s_{g}}\right) t_{i}+\frac{t_{i}}{s_{g}} \int_{0}^{t_{i}} t f_{t}(t) d t\right)\right) \\
\stackrel{(b)}{=} \frac{P_{\Xi_{2}}}{S} \int_{0}^{\bar{d}_{s g}} \bar{d}_{i}\left(\bar{d}_{i}-\frac{\bar{d}_{i}^{2}}{2 S s_{g}}\right) f_{\bar{d}}(\bar{d}) d \bar{d}
\end{aligned}
$$

where $P_{\Xi_{2}}=1-P_{\Xi_{1}}$ is the probability that any SC belongs to $\Xi_{2}$, the first term and the second term in (a) represent that $\mathcal{T}_{0}$ misses any SC of $\Xi_{2}$ completely with probability $1-\frac{t_{i}}{s_{g}}$ and partly from 0 to $t_{i}$ with probability $\frac{t_{i}}{s_{g}}$ respectively, and (b) follows from $t_{i}=\frac{\bar{d}_{i}}{S}$. The expected value of the time that $\mathcal{T}_{0}$ misses during its movement can be obtained as:

$$
\mathbb{E}\left[T_{\text {loss }}\right]=\mathbb{E}\left[N_{s c}\right]\left(\mathbb{E}\left[T_{\Xi_{1}}\right]+\mathbb{E}\left[T_{\Xi_{2}}\right]\right)
$$

\section{ENERGY EFFICIENCY}

The energy efficiency in the uplink is defined as the total achievable data rate that $\mathcal{T}_{0}$ can achieve during its movement divided by the expected value of the total energy that $\mathcal{T}_{0}$ consumes during the same movement. It is assumed that $\mathcal{T}_{0}$ has traffic to send during its movement. Therefore, the total energy includes both the energy consumed for transmitting traffic in the uplink and the energy consumed for performing IFSs during $M_{X_{0}-X_{1}}$. The energy efficiency is expressed as:

$$
\begin{aligned}
\zeta & =\frac{\mathcal{R}_{T}}{\mathbb{E}\left[E_{T}\right]} \\
& =\frac{\mathcal{R}_{1} \chi_{1}+\mathcal{R}_{2} \chi_{2}}{\mathbb{E}\left[E_{1}\right]+\mathbb{E}\left[E_{2}\right]+\mathbb{E}\left[E_{s}\right]}
\end{aligned}
$$

where $\chi_{1}=\frac{\left(\mathbb{E}\left[T_{T}\right]-\mathbb{E}\left[T_{\text {sc }}\right]+\mathbb{E}\left[T_{\text {loss }}\right]\right)}{\mathbb{E}\left[T_{T}\right]}$ and $\chi_{2}=1-\chi_{1}$ are the fractions of time that $\mathcal{T}_{0}$ camps on the MCs and the SCs respectively, $\mathbb{E}\left[T_{T}\right]=\frac{\mathbb{E}\left[D_{X_{0}-X_{1}}\right]}{S}+T_{p}, \mathcal{R}_{1}$ and $\mathcal{R}_{2}$ represent the average ergodic rates of $\mathcal{T}_{0}$ camps on the MCs and the SCs respectively, and $\mathbb{E}\left[E_{1}\right]$ and $\mathbb{E}\left[E_{2}\right]$ represent the energy consumption needed to transmit the uplink traffic when $\mathcal{T}_{0}$ camps on the MCs and the SCs respectively.

\section{A. Energy Consumption}

The expected value of energy consumption for performing IFSs during $M_{X_{0}-X_{1}}$ can be found as:

$$
\mathbb{E}\left[E_{s}\right]=\frac{p_{s} t_{s}}{s_{g}}\left(\mathbb{E}\left[T_{T}\right]-\mathbb{E}\left[T_{s c}\right]+\mathbb{E}\left[T_{\text {loss }}\right]\right)
$$

The expected number of IFSs that $\mathcal{T}_{0}$ performs during its movement depends on the time that $\mathcal{T}_{0}$ camps on the MCs $\mathbb{E}\left[N_{s}\right]=\frac{1}{s_{g}}\left(\mathbb{E}\left[T_{T}\right]-\mathbb{E}\left[T_{s c}\right]+\mathbb{E}\left[T_{\text {loss }}\right]\right)$. When considering the distance-proportional fractional power control similar to [11], 
the expected energy consumption of $\mathcal{T}_{0}$ when transmitting the traffic to the SCs is expressed as:

$$
\begin{aligned}
\mathbb{E}\left[E_{2}\right] & =\chi_{2} \mathbb{E}\left[T_{T}\right] \int_{0}^{\infty} p_{c} x_{2}^{\alpha_{2} \epsilon} f_{x_{2}}(x) d x \\
& =\chi_{2} \mathbb{E}\left[T_{T}\right] \int_{0}^{R_{s c}} 2 \pi \lambda_{2} p_{c} x_{2}^{\alpha_{2} \epsilon+1} e^{-\pi \lambda_{2} x_{2}^{2}} d x \\
& \stackrel{(a)}{=} p_{c} \chi_{2} \mathbb{E}\left[T_{T}\right]\left(\frac{\operatorname{erf}\left(\sqrt{\pi \lambda_{2}} R_{s c}\right)}{2 \sqrt{\lambda_{2}}}-R_{s c} e^{-\pi \lambda_{2} R_{s c}^{2}}\right)
\end{aligned}
$$

where $p_{c}$ is the baseline transmit power of UEs, $\epsilon$ is the power control factor and takes value in the range $[0,1]$, and $x_{2}$ represents the distance from $\mathcal{T}_{0}$ to its serving SC and it is assumed to have a Rayleigh distribution with PDF $f_{x_{2}}(x)=2 \pi \lambda_{2} x_{2} e^{-\pi \lambda_{2} x_{2}^{2}}$, this assumption holds since the probability of $\mathcal{T}_{0}$ camps on the SCs is independent of the distances to the MCBSs as shown in Eq. (3). (a) is obtained from $\epsilon=0.5, \alpha_{2}=4$ and by using [12, 3.321.5], and $\operatorname{erf}($.$) represents the error function. The expected energy$ consumption of $\mathcal{T}_{0}$ when transmitting the traffic to the MCs is expressed as:

$$
\begin{aligned}
\mathbb{E}\left[E_{1}\right] & =\chi_{1} \mathbb{E}\left[T_{T}\right] \int_{0}^{\infty} p_{c} x_{1}^{\alpha_{1} \epsilon} f_{x_{1}}(x) d x \\
& =\chi_{1} \mathbb{E}\left[T_{T}\right] \int_{0}^{\infty} 2 \pi \lambda_{1} p_{c} x_{1}^{\alpha_{1} \epsilon+1} e^{-\pi \lambda_{1} x_{1}^{2}} d x \\
& \stackrel{(a)}{=} \frac{2 p_{c} \chi_{1} \mathbb{E}\left[T_{T}\right]\left(\alpha_{1} \epsilon+1\right) !}{\left(\pi \lambda_{1}\right)^{\alpha_{1} \epsilon+1}}
\end{aligned}
$$

where $x_{1}$ represents the distance from $\mathcal{T}_{0}$ to its serving macro cell and it is assumed to have a Rayleigh distribution, $f_{x_{1}}(x)=$ $2 \pi \lambda_{1} x_{1} e^{-\pi \lambda_{1} x_{1}^{2}}$ is the PDF of $x_{1}$ and (a) is obtained by using $[12,3.351 .3]$.

\section{B. Achievable Rate}

The received signal to interference plus noise ratio (SINR) from $\mathcal{T}_{0}$ at its serving BS from $k$ th tier can be expressed as:

$$
S I N R_{k}=\frac{p_{c} g_{0} P L_{k} x_{k}^{\alpha_{k}(\epsilon-1)}}{\sigma^{2}+\sum_{i \in \Phi_{k}}\left(\hat{z}_{i}^{\alpha_{k}}\right)^{\epsilon} p_{c} h P L_{k} z_{i}^{-\alpha_{k}}}
$$

where $g_{0}$ and $h$ are the channel gain between the serving cell and $\mathcal{T}_{0}$ and interferer terminals respectively, and they are assumed to be exponentially distributed with unity mean, $\sigma^{2}$ is the additive noise power, $\hat{z}$ is the distance between an interferer terminal in uplink and its serving cell, and $z$ is the distance between the $\mathcal{T}_{0}$ 's serving BS and the interferer terminal.

The average achievable rate of $\mathcal{T}_{0}$ associated to the second tier $\mathcal{R}_{2}$ can be expressed similar to [13] as:

$$
\mathcal{R}_{2}=\int_{0}^{\infty} \mathbb{E}_{S I N R_{2}}\left[\ln \left(1+\operatorname{SINR}_{2}\left(x_{2}\right)\right)\right] f_{x_{2}}(x) d x
$$

where $\mathbb{E}_{S I N R_{2}}\left[\ln \left(1+S I N R_{2}\left(x_{2}\right)\right)\right]$ is obtained as:

$$
\begin{aligned}
& =\int_{0}^{\infty} \mathbb{P}\left[\ln \left(1+S I N R_{2}\left(x_{2}\right)\right)>\tau\right] d \tau \\
& =\int_{0}^{\infty} \mathbb{P}\left[\ln \left(g_{0}>\frac{x_{2}^{\alpha_{2}(\epsilon-1)}\left(\sigma^{2}+I_{2}\right)\left(\mathrm{e}^{\tau}-1\right)}{P L_{2} p_{c}}\right)\right] d \tau \\
& =\int_{0}^{\infty} \exp \left(-\frac{x_{2}^{\alpha_{2}(\epsilon-1)} \sigma^{2}\left(\mathrm{e}^{\tau}-1\right)}{p_{c} P L_{2}}\right) \mathscr{L}_{I_{2}}\left(\frac{x_{2}^{\alpha_{2}}\left(\mathrm{e}^{\tau}-1\right)}{p_{c} P L_{2}}\right) d \tau
\end{aligned}
$$

where $\mathscr{L}_{I_{2}}($.$) is the Laplace transform of the cumulative$ interference from terminals in uplink. $\mathscr{L}_{I_{2}}(s)$ can be obtained as:

$$
\begin{aligned}
& =\mathbb{E}_{I_{2}}\left[\mathrm{e}^{-s I_{2}}\right] \\
& =\mathbb{E}_{\Phi_{2}, \hat{z}, h}\left[\exp \left(-s \sum_{i \in \Phi_{2}} p_{c}\left(\hat{z}_{i}^{\alpha_{2}}\right)^{\epsilon} h_{i} z_{i}^{-\alpha_{2}}\right)\right] \\
& \stackrel{(a)}{=} \mathbb{E}_{\Phi_{2}, \hat{z}}\left[\prod_{i \in \Phi_{2}} \frac{1}{1+s\left(\hat{z}_{i}^{\alpha_{2}}\right)^{\epsilon} p_{c} z^{-\alpha_{2}}}\right] \\
& \stackrel{(b)}{=} \exp \left(-2 \pi \lambda_{2} \int_{x_{2}}^{\infty}\left(1-\mathbb{E}_{\hat{z}}\left[\frac{1}{1+s\left(\hat{z}_{i}^{\alpha_{2}}\right){ }^{\epsilon} p_{c} z^{-\alpha_{2}}}\right]\right) z d z\right) \\
& \stackrel{(c)}{=} \exp \left(-2 \pi \lambda_{2} \mathbb{E}_{\hat{z}}\left[\int_{x_{2}}^{\infty} \frac{z}{1+G^{-1} z^{\alpha_{2}}} d z\right]\right) \\
& \stackrel{(d)}{=} \exp \left(\frac{-2 \pi \lambda_{2}}{\alpha_{2}} \mathbb{E}_{\hat{z}}\left[G^{\frac{2}{\alpha_{2}}} B\left(\frac{2}{\alpha_{2}}, 1-\frac{2}{\alpha_{2}}\right)\right]\right)
\end{aligned}
$$

where (a) follows from the fact that $h \sim \exp (1)$, (b) follows from the probability generating functional (PGFL) of the PPP [10] and the integration limits are from $x_{2}$ to $\infty$ since the distance between the serving SCBS and closest interferer terminal is $x_{2}$, and (c) follows from changing the order of the integration and $G=s\left(\hat{z}^{\alpha_{2}}\right)^{\epsilon} p_{c} P L_{2}$. Since $x_{2}$ is very small in comparison to the area of the system, the integration limits are assumed to be from 0 to $\infty$. (d) follows from [12, 3.194.3] after substituting $v=z^{\alpha_{2}}$ and $d z=\frac{z^{1-\alpha_{2}} d v}{\alpha_{2}}$, and $B($.$) denotes the Beta function. For tractability, \hat{z}$ is assumed to be Rayleigh distribution $f_{\hat{z}}(\hat{z})=2 \pi \lambda_{2} \hat{z} \mathrm{e}^{-\pi \lambda_{2} \hat{z}^{2}}$. Thereby $\mathscr{L}_{I_{2}}(s)$ becomes:

$$
\begin{aligned}
& \mathscr{L}_{I_{2}}(s)=\exp \left(-\frac{2 \pi \lambda_{2} x_{2}^{2}\left(e^{\tau}-1\right)^{\frac{2}{\alpha_{2}}}}{\alpha_{2}} B\left(\frac{2}{\alpha_{2}}, 1-\frac{2}{\alpha_{2}}\right)\right. \\
&\left.\int_{0}^{\infty} \hat{z}^{2 \epsilon} f_{\hat{z}}(\hat{z})\right) \\
&=\exp \left(-\frac{\left(2 \pi \lambda_{2} x_{2}\right)^{2}\left(e^{\tau}-1\right)^{\frac{2}{\alpha_{2}}}}{\alpha_{2}} B\left(\frac{2}{\alpha_{2}}, 1-\frac{2}{\alpha_{2}}\right)\right. \\
&\left.\int_{0}^{R_{s c}} \hat{z}^{2 \epsilon+1} \exp \left(-\pi \lambda \hat{z}^{2}\right) d \hat{z}\right)
\end{aligned}
$$

when considering a special case $\left(\sigma^{2}=0\right.$ and $\left.\epsilon=0.5\right)$, and using [12, 3.321.5], $\mathcal{R}_{2}$ becomes:

$$
\mathcal{R}_{2}=\int_{0}^{\infty} \frac{d \tau}{1+C \frac{2\left(e^{\tau}-1\right)^{\frac{2}{\alpha_{2}}}}{\alpha_{2}} B\left(\frac{2}{\alpha_{2}}, 1-\frac{2}{\alpha_{2}}\right)}
$$


where $C=\left(\frac{\operatorname{erf}\left(\sqrt{\pi \lambda_{2}} R_{s c}\right)}{2 \sqrt{\lambda_{2}}}-R_{s c} e^{-\pi \lambda_{2} R_{s c}^{2}}\right)$. Similar way, $\mathcal{R}_{1}$ can be obtained as shown:

$$
\mathcal{R}_{1}=\int_{0}^{\infty} \mathbb{E}_{S I N R_{1}}\left[\ln \left(1+\operatorname{SINR}_{1}\left(x_{1}\right)\right)\right] f_{x_{1}}(x) d x
$$

where $S I N R_{1}$ is the received SINR from the first tier and $\mathbb{E}_{S I N R_{1}}\left[\ln \left(1+S I N R_{1}\right)\right]$ is obtained as:

$$
\begin{aligned}
& =\int_{0}^{\infty} \mathbb{P}\left[\ln \left(1+S I N R_{1}\left(x_{1}\right)\right)>\tau\right] d \tau \\
& =\int_{0}^{\infty} \mathbb{P}\left[\ln \left(g_{0}>\frac{x_{1}^{\alpha_{1}(\epsilon-1)}\left(\sigma^{2}+I_{1}\right)\left(\mathrm{e}^{\tau}-1\right)}{P L_{1} p_{c}}\right)\right] d \tau \\
& =\int_{0}^{\infty} \exp \left(-\frac{x_{1}^{\alpha_{1}(\epsilon-1)} \sigma^{2}\left(\mathrm{e}^{\tau}-1\right)}{p_{c} P L_{1}}\right) \mathscr{L}_{I_{1}}\left(\frac{x_{1}^{\alpha_{1}}\left(\mathrm{e}^{\tau}-1\right)}{p_{c} P L_{1}}\right) d \tau
\end{aligned}
$$

where $\mathscr{L}_{I_{1}}($.$) is the Laplace transform of the cumulative$ interference from terminals of other MCs. $\mathscr{L}_{I_{1}}(s)$ can be obtained similar to Eq. (20) as shown:

$$
\begin{array}{r}
\mathscr{L}_{I_{1}}(s)=\exp \left(-\frac{2 \pi \lambda_{1} x_{1}^{2}\left(e^{\tau}-1\right)^{\frac{2}{\alpha_{1}}}}{\alpha_{1}} B\left(\frac{2}{\alpha_{1}}, 1-\frac{2}{\alpha_{1}}\right)\right. \\
\left.\int_{0}^{\infty} \hat{z}^{2 \epsilon} f_{\hat{z}}(\hat{z})\right) \\
\stackrel{(a)}{=} \exp \left(-2\left(\pi \lambda_{1}\right)^{1-\epsilon} x_{1}^{2}\left(e^{\tau}-1\right)^{\frac{2}{\alpha_{1}}} B\left(\frac{2}{\alpha_{1}}, 1-\frac{2}{\alpha_{1}}\right)\right. \\
\Gamma(\epsilon+1))
\end{array}
$$

where (a) is found from [12, 3.326.2], $\Gamma$ (.) denotes the Gamma function. When $\sigma^{2}=0, \mathcal{R}_{1}$ becomes:

$$
\mathcal{R}_{1}=\int_{0}^{\infty} \frac{d \tau}{2+4\left(\pi \lambda_{1}\right)^{-\epsilon} B\left(\frac{2}{\alpha_{1}}, 1-\frac{2}{\alpha_{1}}\right) \Gamma(\epsilon+1)\left(e^{\tau}-1\right)^{\frac{2}{\alpha_{1}}}}
$$

\section{OPTIMUM INTER-FreQUENCY SCAN RATE}

In this section, the optimum value of IFS rate that achieves the best energy efficiency is obtained. Previously, it was shown that the energy efficiency is a rational function of $s_{g}$, SCs' density, terminals' speed, the coverage of the SCs and other system parameters. Therefore, the optimum value of IFS rate depends on all other system parameters. The energy efficiency in Eq. (13) can be rewritten as shown:

$$
\zeta=\frac{K_{1}+K_{2} s_{g}}{K_{3}+K_{4} s_{g}+K_{5} s_{g}^{-1}}
$$

where $K_{1}=\mathcal{R}_{1}\left(1-\frac{\mathbb{E}\left[T_{s c}\right]}{\mathbb{E}\left[T_{T}\right]}\right)+\mathcal{R}_{2} \frac{\mathbb{E}\left[T_{s c}\right]}{\mathbb{E}\left[T_{T}\right]}, K_{2}=\frac{\mathbb{E}\left[N_{s c}\right]}{2 \mathbb{E}\left[T_{T}\right]}\left(\mathcal{R}_{1}-\right.$ $\left.\mathcal{R}_{2}\right), \quad K_{3}=\left(\mathbb{E}\left[T_{T}\right]-\mathbb{E}\left[T_{s c}\right]\right) \mathbb{E}\left[p_{1}\right]+\mathbb{E}\left[T_{s c}\right] \mathbb{E}\left[p_{2}\right]+$ $0.5 p_{m} t_{m} \mathbb{E}\left[N_{s c}\right], K_{4}=0.5 \mathbb{E}\left[N_{s c}\right]\left(\mathbb{E}\left[p_{1}\right]-\mathbb{E}\left[p_{2}\right]\right)$, and $K_{5}=$ $p_{m} t_{m}\left(\mathbb{E}\left[T_{T}\right]-\mathbb{E}\left[T_{s c}\right]\right) . \mathbb{E}\left[p_{1}\right]$ and $\mathbb{E}\left[p_{2}\right]$ are the expected value of the uplink transmit power when $\mathcal{T}_{0}$ is connected to the MCs and the SCs respectively, and can be obtained similar to Eq. (16) and Eq. (15). The optimum value of $s_{g}$ that maximize the energy efficiency can be found by setting the derivative $\frac{d \zeta}{d s_{g}}=0$. By considering some simplifications $\frac{d \zeta}{d s_{g}}$ is obtained $d s_{g}$
as:

$$
\frac{d \zeta}{d s_{g}}=\frac{K_{1}\left(K_{5}-K_{4} s_{g}^{2}\right)+K_{2} s_{g}\left(K_{3} s_{g}+2 K_{5}\right)}{\left(s_{g}\left(K_{3}+K_{4} s_{g}+K_{5}\right)\right)^{2}}
$$

By setting $\frac{d \zeta}{d s_{g}}=0$, solving the below quadratic equation will give the optimum value of $s_{g}$ :

$$
\left(K_{2} K_{3}-K_{1} K_{4}\right) s_{g}^{2}+2 K_{2} K_{5} s_{g}+K_{1} K_{5}=0
$$

The optimum value of $s_{g}$ is obtained by using the quadratic function as shown below:

$$
s_{g}=\frac{-2 K_{2} K_{5}+\sqrt{\left(2 K_{2} K_{5}\right)^{2}-4\left(K_{2} K_{3}-K_{1} K_{4}\right) K_{1} K_{5}}}{2\left(K_{2} K_{3}-K_{1} K_{4}\right)}
$$

\section{NUMERICAL RESULTS}

In this section, we show the effect of IFS on the system performance in a two-tier HetNet. Fig. 3 shows that the uplink average achievable rate increases significantly when the terminals perform IFS more frequently (low value of $s_{g}$ ). It is also shown that the time missed by the terminals is reduced when the value of $s_{g}$ is small. For instance, when $s_{g}=1$, the UEs exploit more time connecting to the second tier. This graph also shows that terminals miss the coverage of SCs significantly or completely when large values of $s_{g}$ are considered (e.g. 100). As a result, these terminals will only be served by the first tier. However, performing IFSs more frequently (e.g. $s_{g}=1$ ) will cause high energy consumption as the terminals consume more power when carrying out more IFSs during the same time period. In Fig. 4, the effect of $s_{g}$ on the energy efficiency is shown without considering the energy consumption of the SC detection process. It is shown that decreasing the value of $s_{g}$ for any SCs' density can improve the energy efficiency in the network. When the energy consumption for SC detection process is included, adopting different values of $s_{g}$ for different SCs' densities is essential to achieve a good trade-off between the total energy consumption and offloading loss as show in Fig. 5. For instance, when the density is high, $\lambda=80$, IFS with $s_{g}$ as small as 8 is required for the best system performance. Fig. 5 also shows that low dense SCs networks require higher values of $s_{g}$ in order to reach the best energy efficiency.

Fig. 6 shows the optimum value of $s_{g}$ for different values of SCs' density and the SC transmit power. The optimum value of $s_{g}$ decreases when the density of SCs increases for the same transmit power. This is because increasing the SCs' density will provide more offloading opportunities and more frequent scans could improve the system performance. In less dense SC network, less frequent scans will save the power at the terminals' side and achieve the best performance. Since increasing the SCs' transmit power will maximize the footage of the SCs, as a result more offloading opportunities increases in the network. Therefore, Fig. 6 also shows that higher transmit power requires smaller values of $s_{g}$ to achieve the best energy efficiency. Interestingly, the optimum values 
of IFS rate in the high transmit power SC network are larger than the optimum values of IFS rate in the low transmit power SC network when the density of SCs is low $\left(\lambda_{2} \leq 8\right)$.

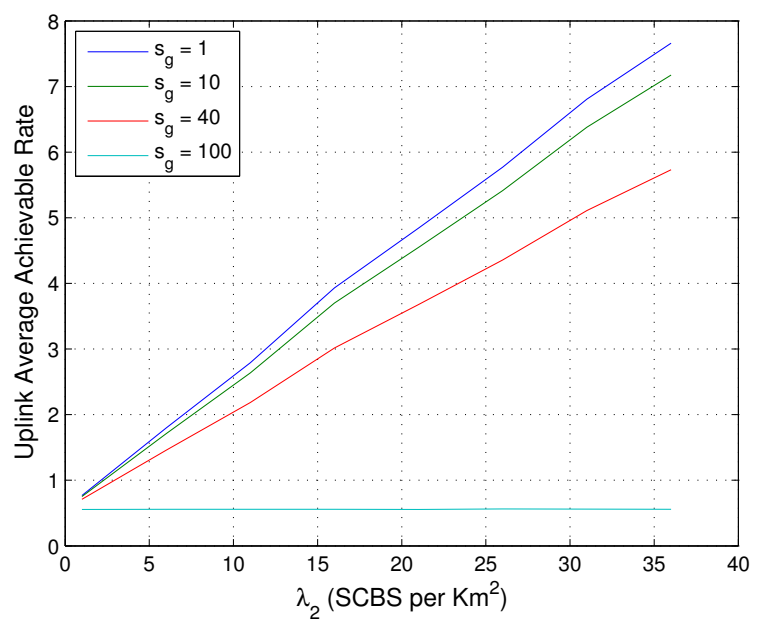

Fig. 3. $\alpha_{2}=\alpha_{1}=4, F_{2}=10 \mathrm{GHz}, F_{1}=2 \mathrm{GHz}, S=5 \mathrm{~km} / \mathrm{h}, \epsilon=0.5$, $\rho_{0}=-90 \mathrm{dBm}, \lambda_{1}=0.5$ and $\mathbb{E}\left[E_{s}\right]=\mathbb{E}\left[N_{m}\right] 2.25 m J$.

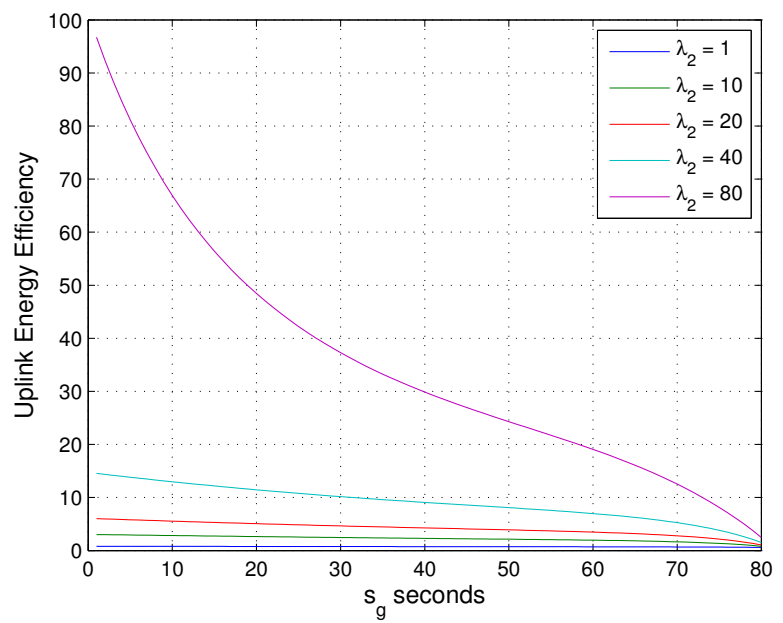

Fig. 4. $\alpha_{2}=\alpha_{1}=4, F_{2}=10 \mathrm{GHz}, F_{1}=2 \mathrm{GHz}, S=5 \mathrm{~km} / \mathrm{h}, \epsilon=0.5$, $\rho_{0}=-90 \mathrm{dBm}, \lambda_{1}=0.5$ and $\mathbb{E}\left[E_{s}\right]=\mathbb{E}\left[N_{m}\right] 2.25 \mathrm{~mJ}$.

\section{CONCLUSION}

In this paper, we presented a framework to model and evaluate the SC detection process and its impact in an interfrequency HetNet, where the uplink energy efficiency is derived as a performance matric. The optimum IFS rate that achieves the best system performance was also derived by taking into consideration the impact of the offloading loss and other system parameters such as the density and transmit power of SCs and the power control. The results show that the smallest value of $s_{g}$ can achieve the best system performance when the energy consumption for the SC detection is ignored

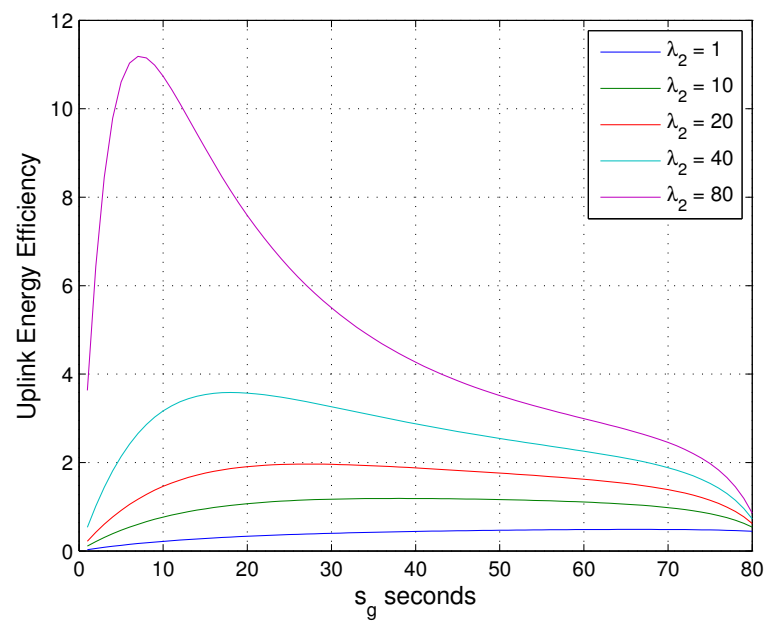

Fig. 5. $\alpha_{2}=\alpha_{1}=4, F_{2}=10 \mathrm{GHz}, F_{1}=2 \mathrm{GHz}, S=5 \mathrm{~km} / \mathrm{h}, \epsilon=0.5$, $\rho_{0}=-90 \mathrm{dBm}, \lambda_{1}=0.5$ and $\mathbb{E}\left[E_{s}\right]=\mathbb{E}\left[N_{m}\right] 2.25 m J$.

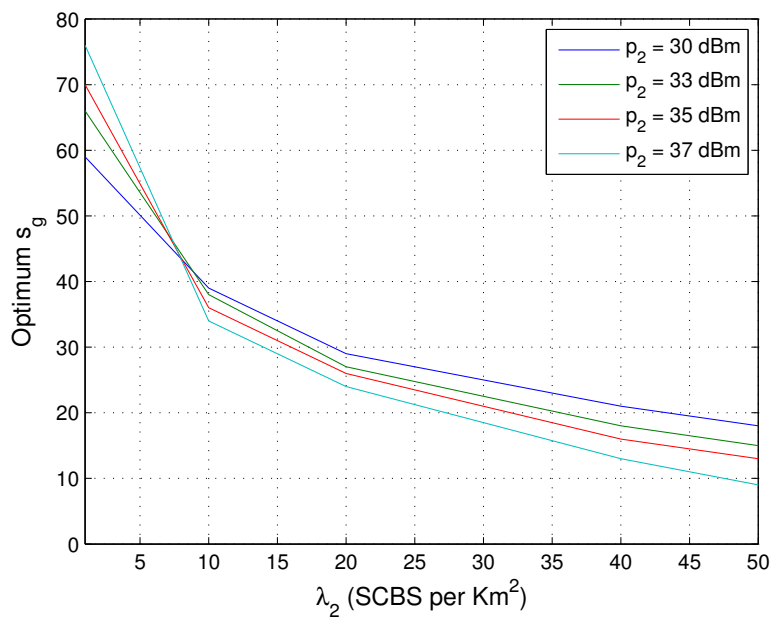

Fig. 6. $\alpha_{2}=\alpha_{1}=4, F_{2}=10 \mathrm{GHz}, F_{1}=2 \mathrm{GHz}, S=5 \mathrm{~km} / \mathrm{h}, \epsilon=0.5$, $\rho_{0}=-90 \mathrm{dBm}, \lambda_{1}=0.5$ and $\mathbb{E}\left[E_{s}\right]=\mathbb{E}\left[N_{m}\right] 2.25 \mathrm{~mJ}$.

in the energy efficiency equation. However, when the energy consumption for SC detection is taken into account, the different values of $s_{g}$ have a great impact on system performance. The results also show that the best system performance can be achieved and the trade-off in the SC detection is minimized when small values of $s_{g}$ are adopted in a dense SCs' network, and when high values of $s_{g}$ are considered in a sparse SCs' network.

\section{REFERENCES}

[1] 3GPP TS 36.331 ver 13.1.0 Release 13, "Technical Specification Group Radio Access Network; Evolved Universal Terrestrial Radio Access (EUTRA)," 32016.

[2] A. Prasad, O. Tirkkonen, P. Lunden, O. N. Yilmaz, L. Dalsgaard, and C. Wijting, "Energy-Efficient Inter-Frequency Small Cell Discovery Techniques for LTE-Advanced Heterogeneous Network Deployments," IEEE Communications Magazine, vol. 51, pp. 72-81, May 2013. 
[3] A. Mahbas, "Double Spectrum Small Cell (DSSC) for Discovering InterFrequency Small Cell in HetNet," IEEE International Conference on Communications (ICC), pp. 3454-3459, 2015.

[4] A. Prasad, P. Lunden, O. Tirkkonen, and C. Wijting, "Mobility State Based Flexible Inter-Frequency Small Cell Discovery for Heterogeneous Networks," 2013 IEEE 24th International Symposium on Personal Indoor and Mobile Radio Communications (PIMRC), pp. 2057 - 2061, 2013.

[5] A. Prasad, P. Lunden, O. Tirkkonen, and C. Wijting, "Enhanced Small Cell Discovery in Heterogeneous Networks Using Optimized RF Fingerprints," 2013 IEEE 24th International Symposium on Personal Indoor and Mobile Radio Communications (PIMRC), pp. 2973 - 2977, 2013.

[6] O. Onireti, A. Imran, M. A. Imran, and R. Tafazolli, "Energy Efficient Inter-Frequency Small Cell Discovery in Heterogeneous Networks," IEEE Transactions on Vehicular Technology, vol. PP, pp. 1 - 13, 2015.

[7] D. B. Johnson and D. A. Maltz, "Dynamic Source Routing in Ad Hoc Wireless Networks," Mobile Computing, pp. 153 - 181, 1996.
[8] S. Shin, U. Lee, F. Dressler, and H. Yoon, "Analysis of Cell Sojourn Time in Heterogeneous Networks With Small Cells," IEEE Communication Letters, vol. 20, pp. 788 - 791, Apr. 2016.

[9] X. Lin, R. K. Ganti, P. J. Fleming, , and J. G. Andrews, "Towards Understanding the Fundamentals of Mobility in Cellular Networks," IEEE Transactions on Wireless Communications, vol. 12, pp. 16861698, Apr. 2013.

[10] D. Stoyan, W. S. Kendall, and J. Mecke, Stochastic Geometry and Its Applications. John Wiley and Sons Ltd., 1995.

[11] A. Mahbas, H. Zhu, and J. Wang, "Unsynchronized Small Cells with a Dynamic TDD System in a Two-Tier HetNet," IEEE 83rd Vehicular Technology Conference: VTC2016-Spring, 2016.

[12] I. Gradshteyn and I. Ryzhik, Table of Integrals, Series, and Products. San Diego, USA: ELSEVIER, 2007.

[13] J. G. Andrews, F. Baccelli, and R. K. Ganti, "A Tractable Approach to Coverage and Rate in Cellular Networks," IEEE Transactions on Communications, vol. 59, pp. 3122-3134, Nov. 2011. 